\title{
Optical mapping of amplitude and phase of excitonic wave functions in a quantum dot system
}

\author{
O. Di Stefano, S. Savasta, G. Pistone, G. Martino, and R. Girlanda \\ Istituto Nazionale per la Fisica della Materia INFM and Dipartimento di Fisica della Materia e Tecnologie Fisiche Avanzate, \\ Università di Messina, Salita Sperone 31, I-98166 Messina, Italy
}

\begin{abstract}
We propose a technique based on a near-field scanning optical microscope, able to measure the spatial variations of both amplitude and phase of the wave functions of the individual eigenstates of a quantum dot system. The proposed scheme is based on a near-field optical microscope working in collection mode combined with a Mach-Zehnder interferometer. We analyze the response function of this device and present microscopic numerical calculations simulating the measurements. These results show that spatially resolved spectroscopy can go beyond measurements of local density of states and open the way to an optical microscopy that, exploiting the coherence properties of light, is able to provide direct and complete quantum-mechanical information on the spatial variations of solid-state mesoscopic quantum eigenfunctions.
\end{abstract}

DOI: 10.1103

\section{INTRODUCTION}

Scanning probe methods are driving the understanding and control over the fundamental building blocks of condensed matter. ${ }^{1}$ The ultimate spatial structure of such blocks is described by quantum-mechanical wave functions. Microscopy which aims to be perfect should be able to provide direct and complete information on this ultimate spatial structure. The scanning tunneling microscope (STM) provides a real-space mapping of the electronic structure at the atomic scale. Specifically, scanning tunneling spectroscopy (STS) measures the differential tunneling conductance $(d I / d V)$ between the STM tip and the sample. $d I / d V$ is to a good approximation proportional to the electronic local density of states (LDOS). ${ }^{2}$ For a system described by discrete electronic wave functions $\psi_{j}(\mathbf{r})$, measurements of the LDOS at a fixed energy $\hbar \omega_{j}$ corresponds to a two-dimensional spatial map of $\left|\psi_{j}(\mathbf{r})\right|^{2}$, i.e., the quantum-mechanical probability density of finding an electron at position $\mathbf{r}$. This possibility has been used to investigate the electronic wave functions of many (eventually nanoconstructed) systems. ${ }^{3}$ However there is more to the wave function than the fact that $\left|\psi_{j}(\mathbf{r})\right|^{2}$ is the probability density. For instance the spatial variation of the phase of the wave function characterizes the probability flux. ${ }^{4}$ Here we propose a technique based on a near-field scanning optical microscope (a derivate of $\mathrm{STM}^{5}$ ), working in collection mode and combined with an optical interferometric technique, able to measure the spatial variations of both amplitude and phase of the wave functions $\psi_{j}(\mathbf{r})$ of the individual eigenstates of a quantum dot system. The results presented here demonstrate that spatially resolved spectroscopy can go beyond measurements of local density of states, thus providing the most complete possible information on the spatial variations of solid-state mesoscopic quantum eigenstates.

Scanning near-field optical microscopy and spectroscopy, which uses optical interaction in the visible or near-infrared range has proven its ability to image optical fields and surface structures at a subwavelength scale. In particular the ability of this kind of optical microscopy and spectroscopy to identify the individual quantum constituents of semiconduc- tor quantum structures has been widely demonstrated. Semiconductor quantum dots (QD's) are artificially fabricated atoms, in which charge carriers are confined in all three dimensions just like electrons in real atoms. Consequently, they exhibit similar properties normally associated with real atoms, such as quantized energy levels and shell structures. Atomic physics progressed rapidly at the beginning of the 20th century, thanks mainly to optical spectroscopy, that has had a decisive influence on the development of our present ideas of atomic and molecular structures. In complete analogy, optical interactions with artificial quantum structures revealed to be of critical importance to provide insight into their electronic structure and dynamics. Moreover, in many cases, coherent optical control and optical manipulation play a fundamental role in the functioning of some proposed devices. ${ }^{6,7}$ QD arrays have distributions in size and shape that lead to inhomogeneous spectral linewidth and averaged data. To avoid this problem these systems are mainly probed by means of techniques of high-resolution spatially resolved spectroscopy and microscopy. In the last few years measurements based on spatially resolved photoluminescence provided direct information on the spatial and energy distribution of light-emitting nanometric dots of semiconductor quantum structures. ${ }^{8-12}$ However spatially resolved photoluminescence provides only an indirect probe of the electronic structure mediated by thermalization effects. Recently Guest et $a l .{ }^{13}$ presented a technique that combines coherent nonlinear spectroscopy with scanning near-field microscopy (SNOM) providing a means to map the exciton (electronhole pair) local density of states (LDOS) of QD systems in analogy to scanning tunneling microscopy (STM) measurements ${ }^{3}$ that mapped out the electronic LDOS.

The analogy between STM and SNOM has been put forward in different contexts and by results from both theoretical analysis and experimental work. ${ }^{14-16}$ However it is worth noting that in STM the eigenmodes of the electron gas near the Fermi level are excited incoherently. SNOM sacrifices the spatial resolution for unmatched spectral resolution and for the possibility of coherent excitation. The technique that we propose exploits the coherence properties of light in order to obtain information on optically active excitonic states be- 
yond LDOS measurements. In the following section, we analyze the response function of the proposed device. Then (Sec. III) we present numerical calculations taking into account the finite spatial resolution. In particular we analyze quantum dots arising from interface fluctuations of GaAs quantum wells (QW's). ${ }^{8}$

\section{THEORY}

In collection-mode SNOM, the sample is usually illuminated by a laser focused onto the sample surface. An optical fiber (waveguide) with a sharp tip is scanned along the sample at subwavelength distance (in the near-field region), and the signal delivered by an optical detector at the end of the fiber is recordered. In experiments done in collection mode the relevant optical field that can be detected by a general near-field setup is given by ${ }^{17,18}$

$$
S_{t o t}(\omega)=A_{b g}(\omega)+S(\omega),
$$

where $A_{b g}(\omega)$ is the background signal largely uniform along the $x-y$ plane and

$$
S(\omega)=A(\omega) \int d z d \mathbf{r} \mathbf{P}(\mathbf{r}, z, \omega) \cdot \mathbf{E}_{\text {out }}(\mathbf{r}, z, \omega),
$$

$A$ is a complex constant depending on the impedance of the material constituting the tip, ${ }^{17} \mathbf{P}(\mathbf{r}, z, \omega)$, is the polarization density of the sample induced by an electromagnetic field $\mathbf{E}_{\text {in }}(\mathbf{r}, z, \omega) . \mathbf{E}_{\text {out }}(\mathbf{r}, z, \omega)$ is the signal mode delivered by a collection-mode SNOM. We have indicated with $\mathbf{r} \equiv(x, y)$ the projection of the position vector on the plane of the QW and with $z$ the coordinate along the growth direction. Following the general linear response theory, the linear macroscopic polarization $\mathbf{P}(\mathbf{r}, z, \omega)$ can be written as ${ }^{19}$

$$
\mathbf{P}(\mathbf{r}, z, \omega)=\int \overleftrightarrow{\boldsymbol{\chi}}\left(\mathbf{r}, z ; \mathbf{r}^{\prime}, z ; \omega\right) \mathbf{E}_{i n}\left(\mathbf{r}^{\prime}, z^{\prime}, \omega\right) d \mathbf{r}^{\prime} d z^{\prime},
$$

where $\overleftrightarrow{\boldsymbol{\chi}}\left(\mathbf{r}, z ; \mathbf{r}^{\prime}, z ; \omega\right)$ is the nonlocal susceptibility tensor. In systems with extended wave functions (such as semiconductors and their quantum structures) nonlocality plays a relevant role. ${ }^{20-23}$ Let us consider the case of naturally occurring quantum dots in quantum wells (see, e.g., Ref. 13). In reasonable good quality QW's the amplitude of the confinement energy fluctuations are typically one order of magnitude smaller than the binding energy of the $1 S$ exciton. In this limit the relative exciton motion described by the effective wave function $\phi_{1 s}\left(\mathbf{r}_{e h}\right)$ may be assumed undistorted by disorder. ${ }^{24}$ In particular, for semiconductor structures described within the usual envelope-function formalism with isotropic electron and hole dispersions, and neglecting fine structure splittings, the susceptibility tensor becomes diagonal with identical elements, ${ }^{22,23}$

$$
\overleftrightarrow{\boldsymbol{\chi}}\left(\mathbf{r}, \mathbf{r}^{\prime}, z, z^{\prime}\right)=\overleftrightarrow{\boldsymbol{\chi}}\left(\mathbf{r}, \mathbf{r}^{\prime}\right) \rho(z) \rho\left(z^{\prime}\right),
$$

where $\rho(z)=\chi_{e}(z) \chi_{h}(z)$ is the product of the electron and hole envelope functions (describing confinement along the growth axis) and

$$
\overleftrightarrow{\boldsymbol{\gamma}}\left(\mathbf{r}, \mathbf{r}^{\prime}\right)=-\frac{\mu^{2}\left|\phi_{1 s}(0)\right|^{2}}{\hbar^{2}} \sum_{n} \frac{\psi_{n}(\mathbf{r}) \psi_{n}^{*}\left(\mathbf{r}^{\prime}\right)}{\omega+i \gamma-\omega_{n}} \overleftrightarrow{\mathbf{1}}
$$

where $\mu$ is the interband dipole moment and $\psi_{n}$ are mesoscopic wave functions describing the excitonic center of mass motion. They are solutions of the following Schrödinger equation:

$$
\hat{H}_{\mathbf{r}} \psi_{n}(\mathbf{r})=\hbar \omega_{n} \psi_{n}(\mathbf{r}),
$$

where $\hat{H}_{\mathbf{r}}$ is the effective Hamiltonian describing in-plane center of mass motion,

$$
\hat{H}_{\mathbf{r}}=-\left(\hbar^{2} / 2 m\right) \nabla^{2}+V(\mathbf{r})+\hbar \omega_{1 s},
$$

with $m$ being the in-plane kinetic mass of the exciton and $V(\mathbf{r})$ the effective disordered potential felt by the exciton as a whole. This effective potential arising from interface fluctuations and alloy disorder can give rise to additional inplane confinement of the exciton. It results from the threedimensional disorder potential (arising from interface fluctuations and alloy disorder) after projection on the valence and conduction subbands and convolution with the $1 \mathrm{~s}$ exciton wavefunction: ${ }^{25}$

$$
V(\mathbf{r})=\int d \mathbf{r}_{e h}\left|\phi_{1 s}\left(\mathbf{r}_{e h}\right)\right|^{2}\left[U_{e}\left(\mathbf{r}_{e h}, \mathbf{r}\right)+U_{h}\left(\mathbf{r}_{e h}, \mathbf{r}\right)\right],
$$

where

$$
U_{e(h)}\left(\mathbf{r}_{e(h)}\right)=\int d z_{e(h)} V_{e(h)}\left(\mathbf{r}_{e(h)}, z_{e(h)}\right) \chi_{e(h)}\left(z_{e(h)}\right),
$$

being $V_{e(h)}\left(\mathbf{r}_{e(h)}, z_{e(h)}\right)$ the 3D disorder potential felt by electrons (holes). We notice that $U_{e}$ and $U_{h}$ in Eq. (8) are obtained from Eq. (9) by simply expressing the in-plane electron (hole) position $\mathbf{r}_{e(h)}$ in terms of the in-plane center of mass $\mathbf{r}$ and relative $\mathbf{r}_{e h}$ positions.

We can now insert Eq. (3) into Eq. (2). Furthermore observing that in the collection mode the input field can be considered constant along the sample, we obtain

$$
S(\omega)=A \int d \mathbf{r} d \mathbf{r}^{\prime} \chi\left(\mathbf{r}, \mathbf{r}^{\prime}, \omega\right) \mathbf{E}_{\text {in }} \cdot \mathbf{E}_{\text {out }}\left(\mathbf{r}^{\prime}, \omega\right)
$$

where

$$
\mathbf{E}_{\text {in/out }}(\mathbf{r}, \omega)=\int \mathbf{E}_{\text {in/out }}(\mathbf{r}, z, \omega) \rho(z) d z .
$$

For the sake of simplicity, we describe the narrow light beam collected by the SNOM tip by a Gaussian electromagneticfield profile,

$$
\mathbf{E}_{\text {in }} \cdot \mathbf{E}_{\text {out }}(\mathbf{r})=\mathcal{F}(\omega) g(\mathbf{r}),
$$

where $g(\mathbf{r})=\exp \left[-\left(x^{2}+y^{2}\right) / \sigma^{2}\right]$. The actual profile of the EM field generated by a SNOM tip is more complex, and a research topic in itself. ${ }^{26}$ However, this simple choice for $g(\mathbf{r})$ is sufficient to highlight the effects of finite spatial resolution. An arbitrary three-dimensional field distribution can however be included in our treatment through Eq. (11). We 
point out that the use of a given profile is valid under weak tip-sample coupling (by sample here we mean the system producing the position dependent signal $S$ ) so that the singlescattering approximation holds. It has been shown that the single-scattering approximation is accurate in near-field optics for a broad class of samples and/or configurations. We also observe that analogously the direct interpretation of the STM signal as a measurement of the electron LDOS of the sample is valid under weak tip-sample coupling.

Using Eqs. (4) and (5), the optical signal [Eq. (10)] (function of the frequency of input light and of the tip position (R) can be written as

$$
\begin{aligned}
S(\mathbf{R}, \omega)= & -\left|\mu \phi_{1 s}(0)\right|^{2} \mathcal{A}(\omega) \sum_{n} \frac{\int d \mathbf{r}^{\prime} \psi_{n}^{*}\left(\mathbf{r}^{\prime}\right)}{\omega+i \gamma-\omega_{n}} \\
& \times \int d \mathbf{r} g(\mathbf{r}-\mathbf{R}) \psi_{n}(\mathbf{r}),
\end{aligned}
$$

where $\mathcal{A}(\omega)=A(\omega) \mathcal{F}(\omega)$. In the limit of point-like detection $[g(\mathbf{r}-\mathbf{R}) \rightarrow \delta(\mathbf{r}-\mathbf{R})]$, we have that

$$
\int d \mathbf{r} g(\mathbf{r}-\mathbf{R}) \psi_{n}(\mathbf{r}) \rightarrow \psi_{n}(\mathbf{R}) .
$$

If in addition the homogeneous broadening $\gamma$ is much lower than the energy difference between the eigenstates of the system (as it usually happens in many quantum dot systems), the spatial variations of the near-field signal at frequency $\omega$ $=\omega_{n}$ acquired at the tip position $\mathbf{R}$ is simply proportional to the excitonic center of mass wave function,

$$
S\left(\mathbf{R}, \omega_{n}\right) \propto \psi_{n}(\mathbf{R}) .
$$

Of course real tips have finite extension, however if the spatial variation of $g(\mathbf{r}-\mathbf{R})$ is much rapid than that of $\psi_{n}(\mathbf{r})$, the spatial variation of $S\left(\mathbf{R}, \omega_{n}\right)$ are expected to contain direct information on the spatial variation $\psi_{n}(\mathbf{R})$. We observe that the combination of global illumination and local collection is essential to achieve this result. On the contrary the local absorption under total illumination ${ }^{21,22}$ is proportional to $\left|\int d \mathbf{r} g(\mathbf{r}-\mathbf{R}) \psi_{n}(\mathbf{r})\right|^{2}$ and maps at best the square modulus of the wave function of the resonant quantum state in complete analogy with STM measurements.

The phase and modulus of $S(\mathbf{R}, \omega)$ can be measured by an interferometric technique recently applied to the collection mode SNOM by Phillips et al. ${ }^{27}$ According to this technique, the optical signal from the local probe does not pass directly to the detection electronics but is combined with a reference signal (a splitted fraction of the input laser beam with frequency $\omega$ and amplitude $L=l e^{i \phi}$ ) as shown in Fig. 1. The two signals are combined by a Mach-Zehnder interferometer operating in the balanced homodyne detection with a phase sensitive element in the upper arm introducing a phase shift $\phi_{p}$. The output signal $W_{t o t}$ in the balanced homodyne detector is given by $^{28}$

$$
W_{t o t}=\left[|L|^{2}-\left|S_{\text {tot }}\right|^{2}\right] \cos \left(\phi_{p}\right)+\left[L^{*} S_{t o t}+S_{t o t}^{*} L\right] \sin \left(\phi_{p}\right) .
$$

Fixing $\phi_{p}=\pi / 2$, the signal detected is

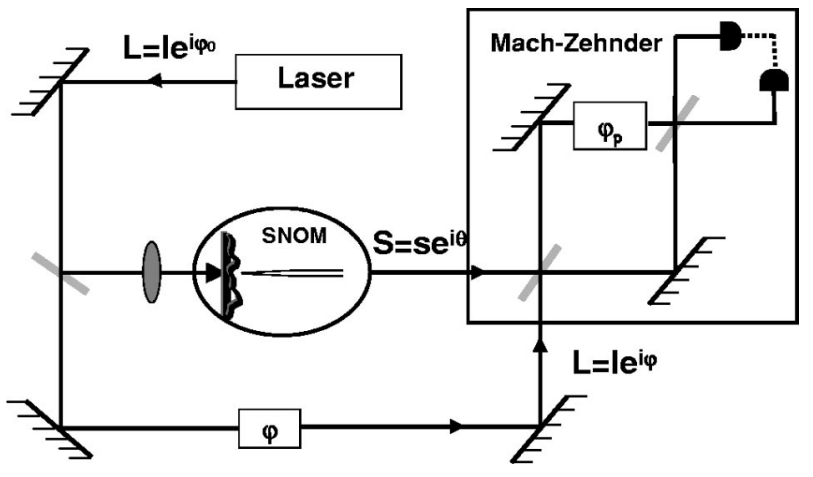

FIG. 1. Schematic representation of the setup able to map quantum eigenfunctions.

$$
W_{t o t}=\left[L^{*} S_{t o t}+S_{t o t}^{*} L\right]=W+W_{b g},
$$

where

$$
W=2 l|S| \cos (\theta-\phi)
$$

and

$$
W_{b g}=2 l\left|A_{b g}\right| \cos \left(\theta_{b g}-\phi\right),
$$

being $\theta_{b g}$ and $\theta$ the phase of $A_{b g}$ and $S$, respectively. We observe that $W_{b g}$ gives a spatially uniform contribution that can be automatically removed by standard techniques. Hence the relevant signal reduces to $W$. We also observe from Eq. (15) that the higher intensity of the output signal is obtained when $\phi=\theta$. Hence, tuning the laser frequency and varying the phase $\phi$ of the splitted laser beam, it is possible to map modulus and phase of the system wave functions. In particular when, tuning the phase $\phi$, the output signal reaches its maximum (at $\phi=\theta_{g}$ ), its value gives the amplitude of the wave function. Thus we have a direct access to the amplitude and phase of $S$ and hence [according to Eqs. (12) and (13)] of the wave function .

We point out that the microscopy proposed here is different from the measurement of the amplitude and phase of Rydberg wave packets performed in Cesium atoms. ${ }^{29}$ In that case one deals with coherently phased superpositions of nondegenerate eigenstates; the amplitude and phase of the coefficients of the superposition being the measured quantities. In contrast our microscopy of eigenfunctions goes inside the spatial variations of individual atomic-like eigenfunctions and it can be considered as complementary to that kind of measurement.

\section{NUMERICAL RESULTS}

Among the most investigated quantum structures are QD systems formed naturally by interface steps in narrow quantum wells. ${ }^{9,10}$ Specifically, the electrons and holes become localized into QD's in regions of the quantum well that are a monolayer wider than the surrounding region and, therefore, have a slightly smaller confinement energy. These well width fluctuations arise from monolayer-high islands at the interfaces which are randomly formed on the growth-interrupted surface. In the following, we present specific calculations for one of these naturally occurring dots. 

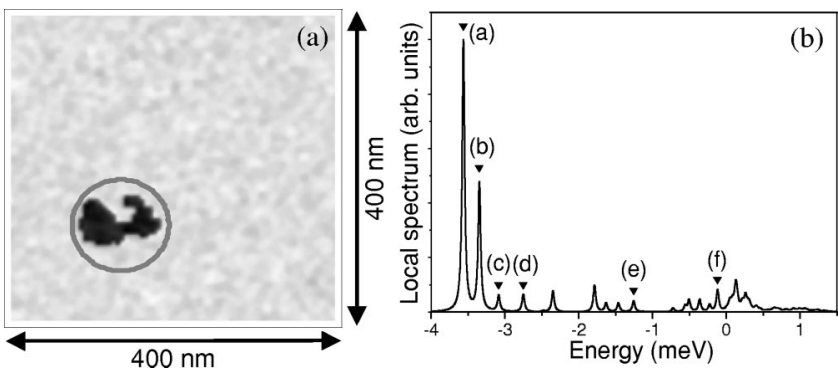

FIG. 2. (a) Specific realization of disorder potential in the region where local spectra have been calculated. The circle specify the location chosen to calculate the local spectrum shown in Fig. 2(b); (b) absorption spectrum under local illumination.

The potentials felt by electrons and holes are assumed to be correlated. In particular [see Eq. (8)], we consider $U_{e}$ $=2 U / 3$ and $U_{h}=U / 3$. We employ a disordered potential $U\left(\mathbf{r}_{e(h)}\right)$ made of two contributions, one step-like contribution of amplitude $-4 \mathrm{meV}$ related to monolayer fluctuations, plus a contribution of smaller amplitude and correlation length due to alloy disorder. This last contribution is modeled as a zero mean, Gauss distributed and spatially correlated process defined by the property $\left\langle U(\mathbf{r}) U\left(\mathbf{r}^{\prime}\right)\right\rangle$ $=v_{0}^{2} e^{-\left|\mathbf{r}-\mathbf{r}^{\prime}\right|^{2} / 2 \xi^{2}}$, where $\langle\cdots\rangle$ denotes ensemble average over random configurations, $v_{0}$ is the width of the energy distribution, and $\xi$ is the correlation length characterizing the potential fluctuations. This widely adopted model ${ }^{30}$ retains the main physical aspects in a very simple way. We adopted $\xi=5 \mathrm{~nm}$ and $v_{0}=0.4 \mathrm{meV}$. We observe that, even if this potential is correlated on very short distances, and even if the first contribution is steplike, the effective potential felt by excitons is rather smooth and correlated over distances given by the exciton Bohr radius [see Eq. (8)]. The resulting realization of the potential felt by excitons is displayed in Fig. 2(a).

Calculations are carried out in real-space mapping on a fine mesh of points the Hamiltonian ${ }^{22} H_{\mathbf{r}}=-\left(\hbar^{2} / 2 m\right) \nabla^{2}$ $+V(\mathbf{r})$, which is then diagonalized in order to obtain eigenvalues and eigenvectors. We adopt an exciton kinetic mass of $m=0.25 m_{0}$ typical for AlAs/GaAs quantum wells. The spectra have been calculated by considering a square region of $0.4 \times 0.4 \mu \mathrm{m}^{2}$ which has been reproduced with a 80 $\times 80$ mesh. Periodic boundary conditions have been adopted. For all the calculated spectra we used a homogeneous broadening $\gamma=20 \mu \mathrm{eV}$. Before calculating the collected signal $S$, in order to get information on the local optical resonances of the system, we calculated absorption spectra under local illumination $(\mathrm{FWHM}=60 \mathrm{~nm}){ }^{22}$ Fig. 2(b) displays one of these spectra obtained with the beam centered at the position indicated by the circle in Figure. 2(a). The spectrum clearly shows sharp distinct peaks characteristic of zero-dimensional exciton states. Figure 3 simulates what can be obtained by the set up shown in Fig. 1 for five different spatial resolutions. In particular it displays $|S| \cos \theta$ for six different values of the laser frequency $\omega$ corresponding to the energy peaks labeled by letters in Fig. 2(b). Each column displays results for a fixed energy and for different spatial resolution (from top to bottom full width at half

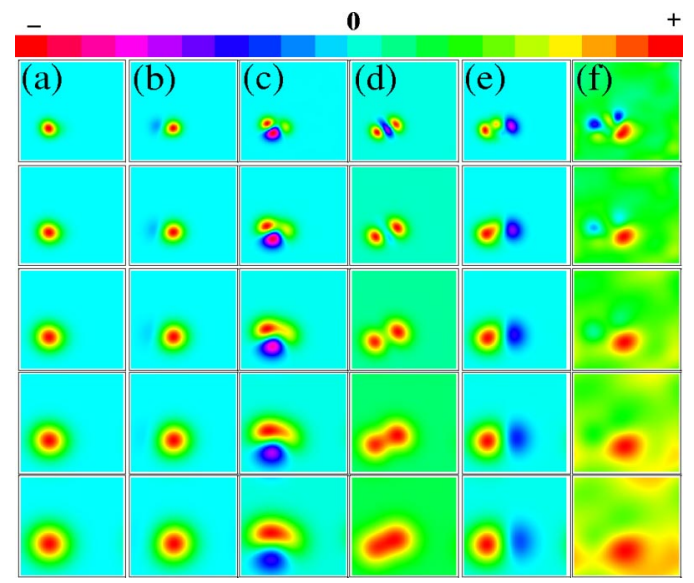

FIG. 3. (a-f) Images displaying $|S| \cos \theta$ for six different values of the laser frequency $\omega$ corresponding to the energy peaks labelled by letters in Fig. 2(b). Each column displays results for a fixed energy and for different spatial resolution. From top to the bottom FWHM is respectively 40, 60, 80, 100, and $120 \mathrm{~nm}$.

maximum (FWHM) of $g(\mathbf{r})$ is $40,60,80,100$ and $120 \mathrm{~nm}$ respectively). As expected, the fundamental state (column $a$ ) has a uniform phase, while the excited states display clear phase variations. We observe that, in the system under study, wave functions can be chosen as real and thus can display phase changes only by $\pi$ (i.e., sign changes of $|S| \cos \theta$ ). In order to check the relationship between the simulated images shown in Fig. 3 and actual wave functions, we display in Fig. 4 the six eigenfunctions corresponding to the energy peaks labeled by letters in Fig. 2(b). We observe that the images displayed in $(\mathrm{a}-\mathrm{e})$ reproduce quite well the modulus and phase variations of the eigenfunctions shown in Fig. 4. The differences between the images are mainly due to cancellation effects originating from the spatial interference of the corresponding quantum state due to the finite resolution of the collecting tip. ${ }^{23,22}$ The comparison also shows noise (see column $(f)$ in Fig. 3) due to tail contributions coming from other states. They appear when the imaged quantum state is not well separated in energy from other states (in this case the quasi-two-dimensional exciton continuum). Figure 3 shows how details are lost when lowering the spatial resolu-

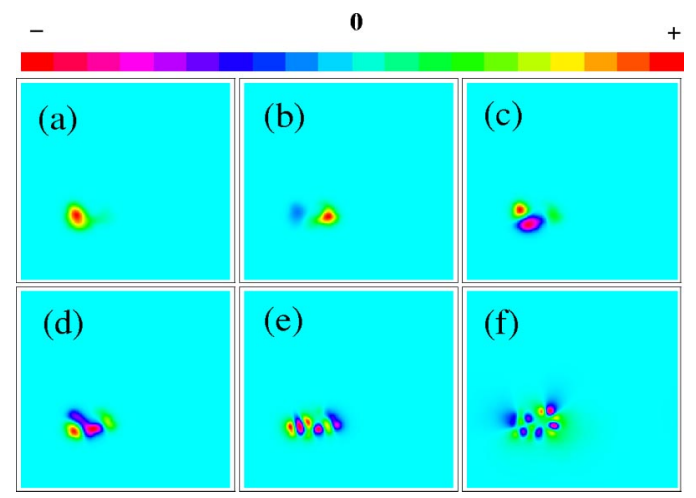

FIG. 4. (a-f) Calculated wavefunctions $\psi(\mathbf{r})$ for the six different values of the laser frequency $\omega$ corresponding to the energy peaks labeled by letters in Fig. 2(b). 
tion. However we observe that also at quite modest resolutions $(\mathrm{FWHM}=100-120 \mathrm{~nm})$ partial information about phase variations survive. We observe that difficulties in achieving high spatial resolutions are mainly due to samples rather than the instrument. In order to obtain samples of good optical quality, the active well with the spontaneously formed dots cannot be much close to the surface. It is the thickness of the barrier above the well (cap layer) which mainly determines the spatial resolution achievable. Recently it has been shown that samples with a cap layer of $20 \mathrm{~nm}$ thickness can provide a sufficiently good quality samples which allows to achieve a spatial resolution of $30 \mathrm{~nm}$.

\section{CONCLUSIONS}

We have shown, by means of theoretical analysis and numerical simulations, that near-field optical microscopy and spectroscopy can go beyond LDOS measurements of scanning tunneling microscopy and spectroscopy, thus achieving the ultimate characterization tool for mesoscopic quantum systems.

In particular we have proposed an experimental setup able to optically map the phase and amplitude of the wave functions of a quantum dot system. Numerical calculations on a GaAs quantum dot displayed in Fig. 3 shows that spatial resolutions available within current near-field techniques suffices. Moreover it is worth noting that the proposed technique allows in addition the separation of resonant photoluminescence always present in these systems from the coherent signal, being the first an incoherent signal, hence largely independent on phase shifts $\phi$. Also the technique provide a direct way to measure the excitonic optical LDOS employing a linear technique hence free from distortions due to the excitation density. Moreover of great interest could be the application of this technique to the real-space analysis of the spatial coherence of quasi-two-dimensional excitons in high quality QW's.

The method proposed here and analyzed can be applied to a large class of optically active two-dimensional mesoscopic quantum systems. Also of great interest would be the possible characterization of local phase changes when applying perturbations as magnetic and electric fields. Finally we believe that this technique would greatly assist the development of nanodevices based on tuning the interaction between individual electronic states.

\section{ACKNOWLEDGMENTS}

We would like to thank E. Cefalì, W. Langbein, and S. Patanè for useful discussions.
${ }^{1}$ Nanometer Scale Science and Technology, Proceedings of the International School of Physics "Enrico Fermi," Course CXLIV, edited by M. Allegrini, N. García, and O. Marti (IOS Press, Amsterdam, 2000).

${ }^{2}$ J. Tersoff and D.R. Hamann, Phys. Rev. B 31, 805 (1985).

${ }^{3}$ See, e.g., M.F. Crommie, C.P. Lutz, and D.M. Eigler, Science 262, 218 (1993); S.G. Lemay, J.W. Janssen, M. van den Hout, M. Mooij, M.J. Bronikowski, P.A. Willis, R.E. Smalley, L.P. Kouwenhoven, and C. Dekker, Nature (London) 412, 617 (2001); T.M. Wallis, N. Nilius, and W. Ho, Phys. Rev. Lett. 89, 236802 (2002).

${ }^{4}$ J.J. Sakurai, Modern Quantum Mechanics (Benjamin/Cummings, California, 1985).

${ }^{5}$ A unified formalism which deals with both STM and SNOM was recently developed by R. Carminati and J.J. Sáenz, Phys. Rev. Lett. 84, 5156 (2000).

${ }^{6}$ A. Imamoglu, D.D. Awschalom, G. Burkard, D.P. DiVincenzo, D. Loss, M. Sherwin, and A. Small, Phys. Rev. Lett. 83, 4204 (1999).

${ }^{7}$ I. D'Amico and F. Rossi, Appl. Phys. Lett. 79, 1676 (2001).

${ }^{8}$ H.F. Hess, E. Betzig, T.D. Harris, L.N. Pfeiffer, and K.W. West, Science 264, 1740 (1994).

${ }^{9}$ D. Gammon, E.S. Snow, and D.S. Katzer, Appl. Phys. Lett. 67, 2391 (1995).

${ }^{10}$ D. Gammon, E.S. Snow, B.V. Shanabrook, D.S. Katzer, and D. Park, Phys. Rev. Lett. 76, 3005 (1996); Science 273, 87 (1996).

${ }^{11}$ Q. Wu, R.D. Grober, D. Gammon, and D.S. Katzer, Phys. Rev. Lett. 83, 2652 (1999).

${ }^{12}$ J. Hasen, L.N. Pfeiffer, A. Pinczuk, S. He, K.W. West, and B.S. Dennis, Nature (London) 390, 54 (1997).

${ }^{13}$ J.R. Guest, T.H. Stievater, Gang Chen, E.A. Tabak, B.G. Orr,
D.G. Steel, D. Gammon, and D.S. Katzer, Science 293, 2224 (2001).

${ }^{14}$ C. Chicanne, T. David, R. Quidant, J.C. Weeber, Y. Lacroute, E. Bourillot, A. Dereux, G. Colas des Francs, and C. Girard, Phys. Rev. Lett. 88, 097402 (2002).

${ }^{15}$ R. Carminati and J.J. Sàenz, Phys. Rev. Lett. 84, 5156 (2000).

${ }^{16}$ G. Colas des Francs, C. Girard, and A. Dereux, J. Chem. Phys. 17, 4659 (2002).

${ }^{17}$ J.-J. Greffet and R. Carminati, Prog. Surf. Sci. 56, 133 (1997).

${ }^{18}$ J.A. Porto, R. Carminati, and J.-J. Greffet, J. Appl. Phys. 88, 4845 (2000).

${ }^{19}$ R. Kubo, J. Phys. Soc. Jpn. 12, 570 (1957).

${ }^{20}$ G. Pistone, S. Savasta, O. Di Stefano, and R. Girlanda, Phys. Rev. B 67, 153305 (2003).

${ }^{21}$ O. Mauritz, G. Goldoni, F. Rossi, and E. Molinari, Phys. Rev. Lett. 82, 847 (1999).

${ }^{22}$ O. Di Stefano, S. Savasta, G. Martino, and R. Girlanda, Phys. Rev. B 62, 11071 (2000); Appl. Phys. Lett. 77, 2804 (2000).

${ }^{23}$ O. Di Stefano, S. Savasta, and R. Girlanda, J. Appl. Phys. 91, 2302 (2002).

${ }^{24}$ R. Zimmermann, Phys. Status Solidi B 173, 129 (1992).

${ }^{25}$ H. Castella and J.W. Wilkins, Phys. Rev. B 58, 16186 (1998).

${ }^{26}$ R. L. Chang, P.K. Wei, W.S. Fann, M.T. Hayashi, and S.H. Lin, J. Appl. Phys. 81, 3369 (1997).

${ }^{27}$ P.L. Phillips, J.C. Knight, J.M. Pottage, G. Kakarantzas, and P.St.J. Russell, Appl. Phys. Lett. 76, 541 (2000).

${ }^{28}$ See e.g., M.O. Scully and M.S. Zubairy, Quantum Optics (Cambridge University Press, Cambridge, 1997).

${ }^{29}$ T.C. Weinacht, J. Ahn, and P.H. Bucksbaum, Phys. Rev. Lett. 80, 5508 (1988).

${ }^{30}$ S. Glutsch, D.S. Chemla, and F. Bechstedt, Phys. Rev. B 54, 11592 (1996). 